\title{
Asimetría cráneo-mandibular de Artibeus lituratus (Chiroptera, Phyllostomidae) en Colombia
}

\author{
Camilo López-Aguirre ${ }^{1} \bowtie$, Jairo Pérez-Torres ${ }^{1}$
}

\section{Cranial and mandibular asymmetry in Artibeus lituratus (Chiroptera, Phyllostomidae) from Colombia}

\begin{abstract}
Analyzing asymmetry in species associated with disturbed environments enables the evaluation of the morphological plasticity of generalistic species and the different evolutionary responses of sexes or populations to environmental or genetic stress. This report is a study of the cranial and mandibular asymmetry of Colombian Artibeus lituratus. This species has a wide distribution and high abundance, but its morphological plasticity remains uncertain. We characterized its presence, fluctuating asymmetry, directional asymmetry and antisymmetry by measuring 11 craneometric traits in 146 adults from different localities. Fluctuating asymmetry was present in all traits; directional asymmetry and antisymmetry in three; and no measurement error in any trait. Females showed more fluctuating asymmetry in the splachnocranium and males in the neurocranium. Traits with functional importance while biting had lower levels of asymmetry and higher similarity. Traits with antisymmetry did not show association while traits with directional asymmetry showed mandibular association. We discuss the relation between the presence of cranial and mandibular asymmetry, with the functional similarity of different traits.
\end{abstract}

Keywords: Stenodermatinae; ecomorphology; craniometry; asymmetry parameter; Neotropics.

Edited by Alberto Acosta四

1. Pontificia Universidad Javeriana, Facultad de Ciencias,

Departamento de Biología, Unidad de Ecología y Sistemática

(UNESIS), Laboratorio de Ecología Funcional, Bogotá, Colombia.

Received: 28-07-2014 Accepted: 22-09-2014

Published on line: 13-10-2014

Citation: López-Aguirre C, Pérez-Torres J (2015) Asimetría cráneomandibular de Artibeus lituratus (Chiroptera, Phyllostomidae) en Colombia. Universitas Scientiarum 20(1): 141-152 doi: 10.11144/Javeriana.SC20-1.acal

Funding: N/A

Electronic supplementary material: Suppl. 1

\section{Introducción}

La asimetría fluctuante (AF) es la desviación aleatoria entre el lado derecho e izquierdo de un plan de simetría bilateral perfecta dentro de una población de organismos (Graham et al. 2010). Se utiliza como indicador de la capacidad de los organismos para enfrentar cambios en el ambiente que impliquen una inestabilidad durante su desarrollo (ID), y refleja así el grado de adaptación del organismo (Knierim et al. 2007). La presencia de AF es la respuesta al estrés, tanto ambiental como genético. El estrés genético representa la condición natural de susceptibilidad de los organismos a presentar AF, mientras que el estrés ambiental representa la presión que ejerce el ambiente en el cual se desarrollan los organismos (Naugler \& Ludman 1996). 
La constitución genética explica la presencia de fenotipos muy marcados de asimetría y de patrones repetitivos de asimetría en caracteres correlacionados (Kellner \& Alford 2003). La concordancia entre los niveles de AF en un grupo de caracteres se explica por la correlación que pueden presentar caracteres similares ontogénica o funcionalmente, de acuerdo con la hipótesis de la integración morfológica (Leamy 1977). Se ha establecido lo que se conoce como parámetros de asimetría, que pueden ser evaluados tanto a nivel individual (Soulé 1967) como a nivel poblacional. Estos permiten analizar la correlación en la forma en que los individuos dentro de una población regulan la respuesta ontogénica a fluctuaciones ambientales (Soulé \& Baker 1968).

La AF se ha usado para evaluar la variabilidad fenotípica dentro de poblaciones silvestres, analizando la relación de esta con el aislamiento geográfico y la variabilidad genética (Zachos et al. 2007, Habel et al. 2012). El aislamiento geográfico afecta directamente el flujo génico de la población, lo que a su vez tiene una relación con la variación morfológica, y ésta puede ser estimada a través de las diferencias en la AF presentes al interior de la población (Habel et al. 2012). La reducción en la variabilidad génica tiene una relación inversa con los niveles de $\mathrm{AF}$, de forma que, a medida que disminuye la heterocigosidad, aumenta la AF (Messier \& Mitton 1996).

La Asimetría Direccional (AD) es un tipo de asimetría en la que todos los individuos dentro de una población mantienen un patrón de asimetría constante. Esta asimetría ha sido caracterizada como un tipo de asimetría natural propia de la biología de la población, la cual puede derivarse tanto de la herencia genética, como de la importancia funcional que adquieran ciertos rasgos con respecto al ambiente en el que se desarrollen (Carter et al. 2009).

La antisimetría (AA) se considera la variación aleatoria del plan de simetría entre los individuos de una población (Graham et al. 2010); su interpretación biológica ha sido debatida, ya que se le han atribuido tanto características hereditarias (Graham et al. 1993b) como de respuesta al estrés (Carter et al. 2009).

La mayoría de estudios de asimetría fluctuante en mamíferos se han centrado en especies raras y con algún grado de amenaza, y se ha subestimado la importancia del estudio de las especies abundantes (Richardson \& Loomis 2009). La mayoría de los estudios en mamíferos silvestres se han centrado en trabajos sobre contaminación (Sonne et al. 2005), comportamiento animal (Mateos et al. 2008), y análisis paisajísticos (Teixeira et al. 2006). Los murciélagos se han usado como modelo de estudio para evaluar la relación entre la $\mathrm{AF}$ y diferentes factores, como la importancia funcional de ciertos caracteres (Gummer \& Brigham 1995), los rangos geográficos de la distribución (Juste et al., 2001a), los patrones de selección sexual (Voigt et al. 2004) y algunos parámetros poblacionales (Juste et al. 2001b).

Diversos estudios de AF han demostrado que evolutivamente se han desarrollado diferencias en la asimetría entre sexos en especies de mamíferos, lo que ha reflejado en algunos casos la presencia de dimorfismo sexual secundario (Gannon et al. 1992). Otros estudios muestran cómo la AF tiene un papel importante en la selección sexual, de forma que los rasgos bajo presión de selección que sean menos asimétricos van a asegurar mayor éxito reproductivo para los individuos (Voigt et al. 2004).

Entender la variación en la AF entre diferentes rasgos de una misma estructura permite evaluar la relación entre la AF y las similitudes funcionales de los rasgos. Con base en esto se ha planteado que la selección natural va a actuar diferencialmente sobre los rasgos dependiendo de su importancia funcional, de forma que los rasgos funcionalmente mas importantes van a tener niveles menores de $\mathrm{AF}$.

El estudio de la plasticidad morfológica y la presencia de $\mathrm{AF}$ en especies con una amplia distribución y que se consideren adaptables permite evaluar a escalas más grandes el efecto del estrés ambiental o genético en la simetría y la respuesta de las especies, así como entender los patrones de asimetría frente a diferencias funcionales entre múltiples rasgos. El estudio de las diferencias en la asimetría entre sexos de una misma especie permite también entender de qué forma los sexos responden diferencialmente a la presión evolutiva como resultado de procesos de dimorfismo sexual o variaciones tróficas (Gannon et al. 1992). 
Artibeus lituratus (Phyllostomidae, Stenodermatinae) es una especie de murciélago frugívora con una amplia distribución geográfica, abundante en ambientes disturbados (Galindo 2004), y se ha usado como especie indicadora de fragmentación de hábitats (Medellín et al. 2000). En Colombia está distribuida en todo el país: se encuentra en sistemas productivos de ganadería (Calonge et al. 2010) y agricultura (OrtegónMartínez \& Pérez-Torres 2007), en zonas de bosque tropical (Estrada-Villegas et al. 2010) y en sistemas urbanos (Alberico et al. 2005). La morfología craneal de esta especie varía respondiendo a fluctuaciones en variables ambientales asociadas a la precipitación en zonas secas (Marchán-Rivadeneira et al. 2012).

En el presente estudio determinamos la presencia de $\mathrm{AF}, \mathrm{AD}$ y AA en cráneos y mandíbulas de $A$. lituratus de diferentes regiones de Colombia respondiendo las siguientes preguntas: (1) ¿La similitud que presenten distintos caracteres craneométricos se relaciona con la asociación e integración entre los diferentes tipos de asimetría?, y (2) ¿Existen diferencias en la asimetría cráneo-mandibular entre machos y hembras?

\section{Materiales y métodos}

\section{Ejemplares analizados}

Se analizaron 146 individuos de la especie $A$. lituratus (75 machos, 63 hembras, 6 sin información) provenientes de 11 departamentos de Colombia (Caldas $[n=3]$, Chocó $[n=3]$, Córdoba $[n=5]$, Cundinamarca $[n=4]$, Huila $[n=47]$, Meta $[n=$ 5], Quindío [ $n=33]$, Risaralda [ $n=3]$, Santander $[n=1]$, Tolima $[n=15]$, Valle del Cauca $[n=18]$ ) que representan cerca de 25\% del área de distribución de la especie en el país. El área de estudio está ubicada entre

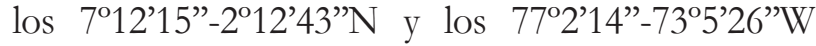
(Figura 1). Todos los especímenes se encuentran depositados en la colección de mamíferos del Museo Javeriano de Historia Natural, Bogotá, Colombia (Supl. 1).

\section{Toma de las imágenes}

Se fotografiaron los cráneos y las mandíbulas con una cámara Nikon D5100 a una resolución de 300 ppp con unas dimensiones de 4928 x 3264 pixeles. Todas las fotografías fueron tomadas por la misma persona, con el mismo lente, y la misma distancia focal

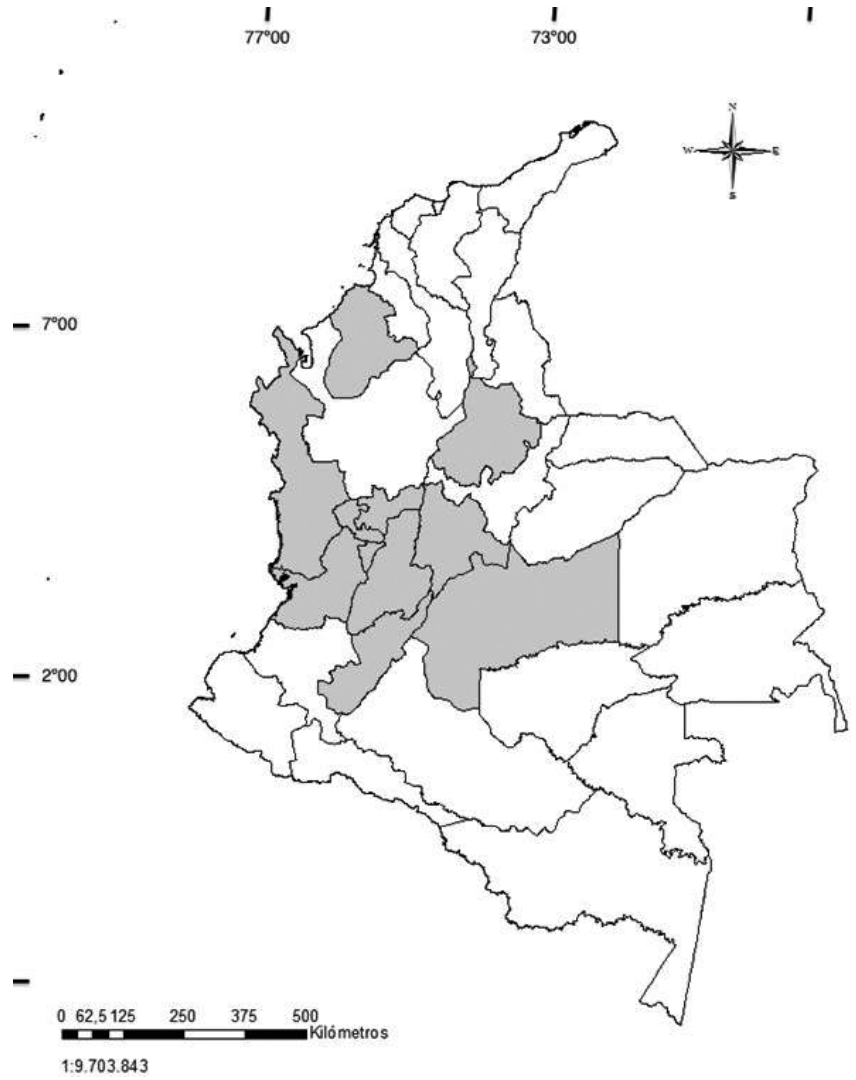

Fig. 1. Área de la distribución de $A$. lituratus representada a partir de las muestras estudiadas en este trabajo (En gris: Caldas, Chocó, Córdoba, Cundinamarca, Huila, Meta, Quindío, Risaralda, Santander, Tolima y Valle del Cauca).

(45 mm), tomando en cuenta lo sugerido por Graham et al. (2010). Para estandarizar la toma de las fotografías (sobre el plano horizontal) se utilizó un soporte para la cámara con el fin de controlar la distancia al objetivo y la iluminación, y se utilizaron soportes independientes para los cráneos y las mandíbulas. El ángulo de inclinación tanto de la cámara como de los cráneos y mandíbulas se controló con un clinómetro para asegurar que estos dos últimos quedaran paralelos.

\section{Medidas craneométricas}

Se registraron 11 medidas craneométricas a partir de las fotografías digitales obtenidas de los cráneos (Figura 2). Estas medidas se escogieron por su importancia como caracteres diagnósticos para distinguir especies del género (Kalko \& Handley 1994). Las medidas fueron tomadas independientemente para cada lado del cráneo, tomando como referencia su eje sagital. 


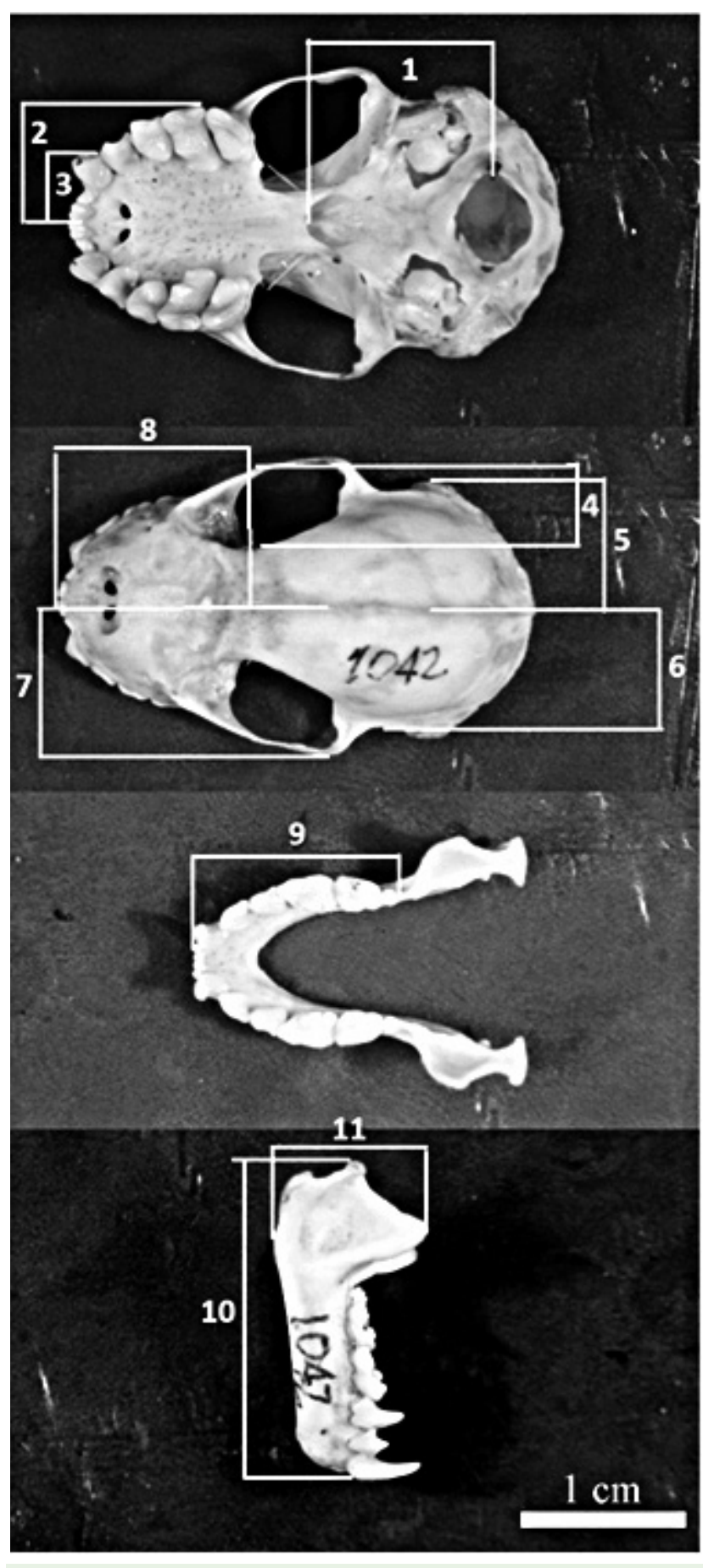

Fig. 2. Medidas craneométricas incluidas para este estudio. Las medidas fueron adaptadas al interés particular del estudio de la asimetría. 1. Longitud postpalatina (LP), 2. Ancho a los molares (AMO), 3. Ancho a los caninos (AC), 4. Ancho interorbital (AI), 5. Ancho al mastoideo (AMA), 6. Ancho de la caja craneal (ACC), 7. Ancho zigomático (AZ), 8. Longitud rostral (LR), 9. Longitud serie dental mandibular (LSD), 10. Longitud de la mandíbula (LM), 11. Altura al proceso coronoide (APC).
Lasvariablesfueronagrupadasconrespectoacriterios morfofuncionales, discriminando el neurocráneo (LP: longitud postpalatina, AI: ancho interorbital, AMA: ancho al mastoideo, ACC: ancho de la caja craneal, y AZ: ancho zigomático) del esplacnocráneo (AMO: ancho a los molares, AC: ancho a los caninos, LR: longitud rostral, LSD: longitud serie dental mandibular, LM: longitud de la mandíbula, y APC: altura al proceso coronoides). Las fotografías fueron procesadas con el programa TpsDig2 (Rohlf 2013) para obtener los valores de las medidas de cada lado del cráneo. Todas las medidas fueron tomadas por el primer autor.

\section{Estimación de asimetrías y error de medida}

Para este estudio se tomó la asimetría como la desviación entre las medidas del lado derecho e izquierdo de una estructura, dentro de un plan de simetría bilateral. Cada tipo de asimetría se definió de la siguiente forma: AF es una desviación aleatoria en la que los valores tienen una distribución normal con media de cero, $\mathrm{AD}$ es una desviación constante en la que los valores tienen una distribución normal con media diferente a cero, y AA es una desviación aleatoria en la que los valores tienen una distribución platicúrtica o bimodal.

Para estimar la presencia de AD y el Error de Medida (EM) se seleccionó aleatoriamente una submuestra de 20 especímenes a los que se les tomaron las medidas dos veces. Con estos datos se realizó un modelo mixto de ANOVA de dos vías tomando medidas repetidas de cada lado para cada variable, y calculando la significancia para la variación interindividual como un indicador de EM. Posteriormente, con los mismos datos se realizó nuevamente un modelo mixto de ANOVA de dos vías con medidas repetidas de cada lado, se tomaron el "lado" (derecho o izquierdo) y la réplica como factores fijos, y la presencia de AD se evaluó con la significancia del factor "lado".

Para evaluar si las AF y AA eran significativas se calculó el valor absoluto de la diferencia entre las medidas de ambos lados de cráneo y mandíbula (derecha-izquierda), y se aplicó el método de análisis de distribuciones estadísticas (Graham et al. 2010). La AF se evaluó con una prueba de Kolmogorov-Smirnov partiendo de una hipótesis de una distribución normal 
de los valores absolutos de asimetría con una media de cero, mientras la AA se evaluó con una prueba de normalidad de Shapiro-Wilks partiendo de una hipótesis de una distribución platicúrtica o bimodal de los valores absolutos de asimetría con una media de cero (Graham et al. 2010). Todos los datos fueron transformados logarítmicamente para evitar efectos de alometría (Graham et al. 2010).

Finalmente, con el fin de analizar los objetivos del estudio, se calculó un índice de AF (1-r) para cada rasgo, para todos los individuos (Windig \& Nylin 2000). Para obtener el valor de $r$ del índice se analizó la relación entre las medidas de ambos lados del cráneo para cada una de las variables mediante la correlación de Spearman con el programa PAST (Hammer et al. 2001).

\section{Asociación morfológica entre variables}

Para responder la pregunta (1) se trató de determinar si había un patrón entre la asociación morfológica y los valores de AF de cada variable, por lo que se agruparon los valores del índice de AF (1-r) de todos los individuos por variables.

Se evaluó el nivel de asociación entre los tres tipos de asimetría en todas las variables mediante un análisis de correlación de Spearman. Finalmente se determinó, mediante un análisis de similitud por conglomerados de Bray-Curtis, qué regiones del cráneo mostraban mayor tendencia a presentar algún tipo de asimetría. Para disminuir la probabilidad de error en las múltiples comparaciones de Spearman, los datos fueron corregidos con una corrección de Bonferroni (Van Dongen et al. 1999). En todos los casos se consideró un nivel de significancia de $\alpha<0.05$.

Para analizar la asociación entre las asimetrías se consideraron todas las variables sin considerar si presentaban más de un tipo de asimetría simultáneamente; esto con el fin de extrapolar estos valores y determinar si el cráneo presentaba regiones con mayor tendencia a presentar cualquier tipo de asimetría ( $\mathrm{AF}, \mathrm{AD}$ y $\mathrm{AA}$ ).

\section{Diferencias entre sexos}

Para responder la pregunta (2) se agruparon por sexos los valores obtenidos previamente del índice de $\mathrm{AF}$ $(1-r)$ de cada rasgo para todos los individuos. Esto se realizó tanto para el cráneo como para la mandíbula. De esta forma se obtuvieron los valores del índice de AF a nivel general y para cada sexo (Windig \& Nylin 2000). Para la interpretación de los patrones de AF no se consideraron las variables que presentaron otro tipo de asimetría o dos tipos de asimetría simultáneamente.

\section{Resultados}

\section{Estimación de asimetrías y error de medida}

A nivel general se encontró significancia para la AF en todas las variables contempladas $(p<0.001)$ (Tabla 1). Respecto a la presencia de error de medida en la submuestra no se encontró significancia de la variación intragrupal para ninguna variable. Por el contrario, sí se evidenciaron diferencias significativas para la presencia de $\mathrm{AD}$ en las variables longitud de la mandíbula (LM; ANOVA: $\mathrm{F}=7.39, \mathrm{n}=20, \mathrm{p}=0.009)$, ancho a los caninos (AC; ANOVA: $\mathrm{F}=47.42, n=20, p=<$ 0.001) y altura al proceso coronoides (APC; ANOVA:

Tabla 1. Significancia de los diferentes tipos de asimetría y del error de medida en las medidas craneométricas. ' $\mathrm{p}<0.05{ }^{*} \mathrm{p}<0.001 \mathrm{y}{ }^{\cdots} \mathrm{p}<0.0001$.

\begin{tabular}{|c|c|c|c|c|}
\hline & AF & AA & $\mathrm{AD}$ & EM \\
\hline LP & $\mathrm{Si}^{\cdots} \cdots$ & No & No & No \\
\hline AMO & $\mathrm{Si}^{\cdots} \cdots$ & $\mathrm{Si}{ }^{\cdot}$ & No & No \\
\hline AC & $\mathrm{Si}^{\cdots} \cdots$ & No & $\mathrm{Si}{ }^{\bullet}$ & No \\
\hline AI & $\mathrm{Si}^{\cdots} \cdots$ & No & No & No \\
\hline AMA & $\mathrm{Si} \cdots$ & No & No & No \\
\hline ACC & $\mathrm{Si} \cdots$ & No & No & No \\
\hline $\mathbf{A Z}$ & $\mathrm{Si}^{\cdots} \cdots$ & $\mathrm{Si} \cdots$ & No & No \\
\hline LR & $\mathrm{Si}{ }^{\cdots}$ & No & No & No \\
\hline LSD & $\mathrm{Si}^{\cdots} \cdots$ & $\mathrm{Si} \cdots$ & No & No \\
\hline LM & $\mathrm{Si}^{\cdots} \cdots$ & No & $\mathrm{Si}{ }^{*}$ & No \\
\hline APC & $\mathrm{Si}^{\cdots} \cdots$ & No & $\mathrm{Si} \cdot$ & No \\
\hline
\end{tabular}


$\mathrm{F}=8.48, n=20, p=0.005)$. Las únicas variables que evidenciaron AA fueron ancho a los molares (AMO; Shapiro-Wilks: $\mathrm{W}=0.97, \mathrm{n}=146, \mathrm{p}=0.0240$ ), ancho zigomático (AZ; Shapiro-Wilks: $\mathrm{W}=0.74, \mathrm{n}=141$, $\mathrm{p}<0.001$ ), y longitud serie dental mandibular (LSD; Shapiro-Wilks: $\mathrm{W}=0.93, \mathrm{n}=140, \mathrm{p}<0.001)$.

\section{Asociación morfológica entre variables}

Todas las variables mostraron algún nivel de AF. Las que mostraron los mayores valores en el índice de asimetría fueron: el ancho de la caja craneal (ACC), el ancho al mastoideo (AMA), y el ancho zigomático (AZ). Las variables que mostraron los menores valores de asimetría fueron: la longitud postpalatina (LP), longitud serie dental mandibular (LSD) y la longitud rostral (LR) (Figura 3).

Todas las variables mostraron correlación positiva y significativa $(\mathrm{p}<0.01)$ con al menos una de las otras variables, excepto la longitud de la serie dental mandibular (LSD), y la longitud postpalatina (LP, Tabla 2). El ancho de la caja craneal (ACC) fue la medida que se correlacionó con más variables, un total de cinco (Tabla 2).

Los valores más altos de correlación se encontraron en el neurocráneo, donde solo se presentó AF. A nivel mandibular se presentaron los valores más bajos de

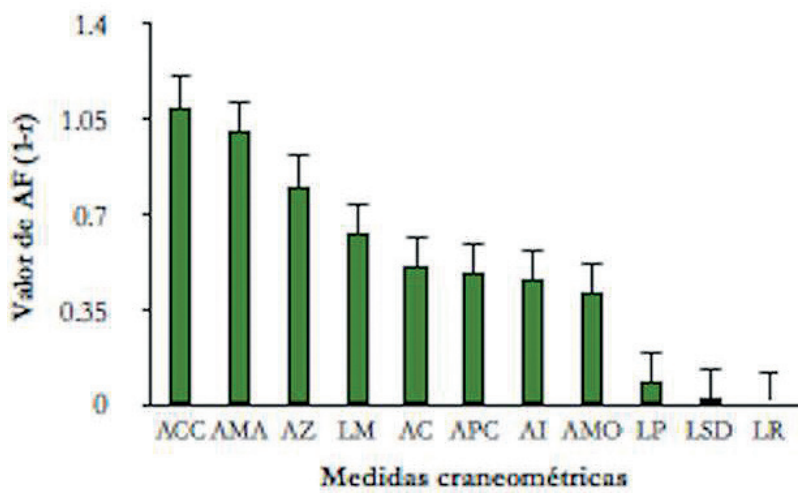

Fig. 3. Valores del índice de AF (1-r) para cada una de las medidas craneométricas.

correlación entre los rasgos con $\mathrm{AD}$, y rasgos del esplacnocráneo como el ancho a los caninos (ACC).

Al considerar todas las variables se observaron dos grupos discretos: el primer grupo constituido por APC, LM, LSD, LR y LP (disimilitud 0.22) que comprenden la región mandibular y el esplacnocráneo. El segundo grupo estuvo compuesto por AZ, ACC y AMA (disimilitud 0.17) que comprenden la región del neurocráneo. Las variables más similares entre sí (disimilitud 0.12) fueron: la longitud serie dental mandibular (LSD) y la longitud rostral (LR); ambas presentaron $\mathrm{AF}$ y están asociadas al esplacnocráneo.

Tabla 2. Correlaciones entre las medidas craneométricas. Debajo de la diagonal se presentan los valores de la correlación de Spearman (ns) y sobre la diagonal, sus correspondientes valores de significancia (p).

\begin{tabular}{ccccccccccccc}
\hline & LP & AMO & AC & AI & AMA & ACC & AZ & LR & LSD & LM & APC \\
\hline LP & & 0.42 & 0.35 & 0.41 & 0.14 & 0.18 & 0.33 & 0.03 & 0.68 & 0.59 & 0.51 \\
AMO & -0.07 & & 0.03 & 0.2 & 0.49 & 0.39 & 0.32 & 0.59 & 0.54 & 0.71 & 0.99 \\
AC & -0.08 & 0.18 & & 0.82 & 0.83 & 0.9 & 0.88 & 0.1 & 0.95 & $<0.001$ & 0.05 \\
AI & 0.07 & 0.11 & 0.02 & & $<0.001$ & $<0.001$ & $<0.001$ & $<0.001$ & 0.45 & 0.84 & 0.07 \\
AMA & 0.12 & -0.06 & 0.02 & 0.59 & & $<0.001$ & $<0.001$ & $<0.001$ & 0.93 & 0.03 & 0.01 \\
ACC & 0.11 & -0.07 & 0.01 & 0.55 & 0.88 & & $<0.001$ & $<0.001$ & 0.98 & 0.02 & $<0.001$ \\
AZ & 0.08 & -0.08 & 0.01 & 0.55 & 0.83 & 0.82 & & $<0.001$ & 0.58 & 0.09 & 0.04 \\
LR & 0.18 & 0.05 & 0.14 & 0.41 & 0.52 & 0.5 & 0.55 & & 0.12 & 0.95 & 0.35 \\
LSD & 0.04 & -0.05 & -0.01 & -0.06 & -0.01 & 0.001 & 0.05 & 0,13 & -0.13 & 0.53 & 0.14 \\
LM & -0.05 & 0.03 & 0.24 & -0.02 & 0.18 & 0.19 & 0.15 & -0.01 & -0.05 & -0.13 & 0.68 & $<0.001$ \\
APC & 0.06 & 0.001 & 0.17 & 0.160 & 0.23 & 0.24 & 0.18 & 0.08 & \\
\hline
\end{tabular}




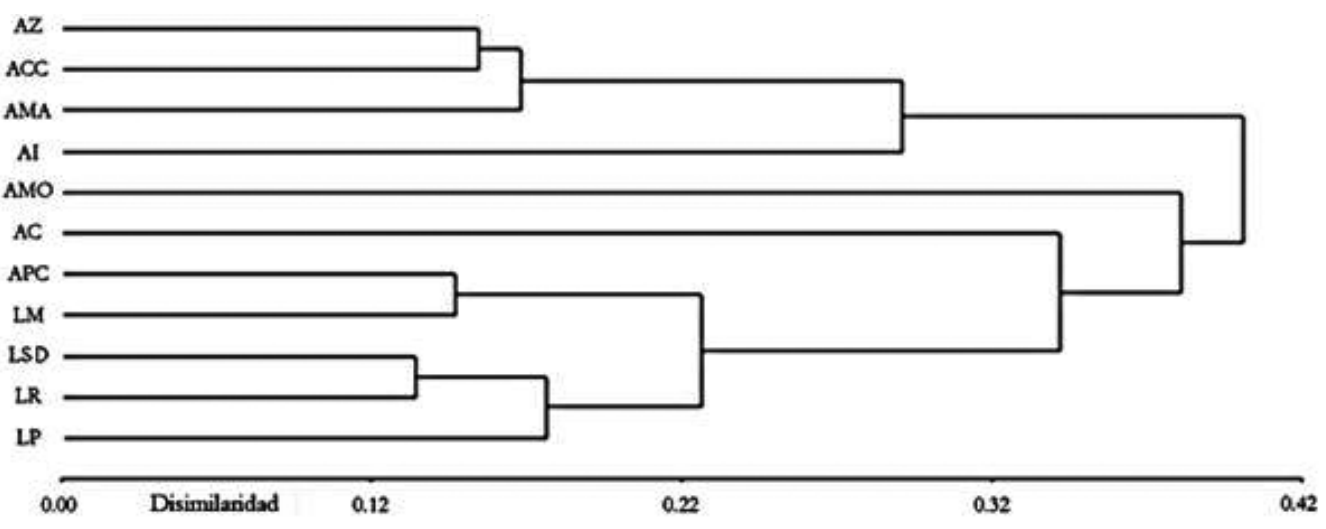

Fig. 4. Cluster del análisis de conglomerados de Bray-Curtis entre todas las variables craneométricas estudiadas.

Las variables con $\mathrm{AD}$ que presentaron una mayor similitud (disimilitud 0.14 ) sobre todo a nivel mandibular fueron la longitud de la mandíbula (LM) y la altura al proceso coronoides (APC). Las variables con mayores niveles de AF que mostraron la mayor similitud fueron AZ y ACC (disimilitud 0.15) (Figura 4).

\section{Diferencias entre sexos}

Al considerar las variables que presentaron más de un tipo de asimetría, se encontró que los machos presentaron más AF a nivel del neurocráneo y las hembras a nivel de esplacnocráneo (Tabla3). Al analizar las variables con solo un tipo de asimetría (AMO, AC, AZ, LSD, LM y APC), las medidas con mayor AF en ambos sexos fueron: ancho de la caja craneal (ACC) y ancho al mastoideo (AMA); la de menor AF fue la longitud rostral (LR). El ancho a los caninos (AC) de las hembras y el ancho interorbital (AI) de los machos fueron las medidas con mayor AF (Tabla 3).

\section{Discusión}

Estimación de asimetrías y error de medida

A nivel mandibular se encontraron más variables con $\mathrm{AD}$, lo cual, de acuerdo con Klingenberg (2008), refleja una integración morfológica en la $\mathrm{AD}$ a nivel mandibular como resultado de una herencia genética. Adicionalmente, la presencia de AA en algunas de las variables craneales puede confirmar, pese a la falta de claridad en el significado biológico de este tipo de asimetría (Graham et al. 1993b), la presencia de un estrés ambiental durante el desarrollo del cráneo en los individuos (Graham et al. 1993a).

La presencia generalizada de AF en todas las medidas muestra que el cráneo es un buen indicador de la variación en el desarrollo morfológico de la especie (Juste et al. 2001a): el desarrollo simétrico de

Tabla 3. Niveles de AF diferenciando entre sexos y entre regiones del cráneo, esplacnocráneo (en blanco) y neurocráneo (en verde).

\begin{tabular}{|c|c|c|c|c|c|c|c|c|c|c|c|c|}
\hline & \multicolumn{6}{|c|}{ NEUROCRANEO } & \multicolumn{6}{|c|}{ ESPLACNOCRANEO } \\
\hline & GRUPO & LP & AI & AMA & ACC & $\mathrm{AZ}$ & LR & LSD & LM & APC & AMO & AC \\
\hline \multirow{3}{*}{$\begin{array}{l}\text { Variables con } \\
\text { más de un tipo } \\
\text { de asimetría. }\end{array}$} & GENERAL & & & & & 0.81 & & 0.03 & 0.63 & 0.49 & 0.41 & \\
\hline & MACHOS & & & & & 0.86 & & 0.02 & 0.65 & 0.45 & 0.38 & \\
\hline & HEMBRAS & & & & & 0.74 & & 0.05 & 0.67 & 0.54 & 0.40 & \\
\hline \multirow{3}{*}{$\begin{array}{l}\text { Variables solo } \\
\text { con asimetría } \\
\text { fluctuante. }\end{array}$} & GENERAL & 0.09 & 0.46 & 1.01 & 0.10 & & 0.01 & & & & & 0.51 \\
\hline & MACHOS & 0.08 & 0.57 & 1.12 & 1.22 & & 0.01 & & & & & 0.42 \\
\hline & HEMBRAS & 0.11 & 0.37 & 0.91 & 1.02 & & 0.01 & & & & & 0.61 \\
\hline
\end{tabular}


esta región se ve afectado por el ambiente, sin importar el estrés genético que presente (Hallgrímsson 1998). Artibeus lituratus es una especie con una distribución geográfica amplia y con una abundancia alta, que muestra una afinidad por los ambientes disturbados (Medellín et al. 2000, Galindo 2004). Sin embargo, su presencia en estos ambientes puede implicar cambios en su desarrollo.

\section{Asociación morfológica entre variables}

La diferencia entre los valores de AF indica que la asimetría a nivel craneal no es uniforme entre todos los rasgos, lo que puede ser una respuesta a la presión ecológica: la funcionalidad de cada rasgo se explica por la ecología de la especie (Gummer \& Brigham 1995). Artibeus lituratus es un frugívoro especializado en el consumo de frutos grandes y duros (Santana \& Dumont 2009), lo que hace que algunas zonas del cráneo asociadas a la mordida presenten una alta importancia funcional. Esto explica que la región rostral sea relativamente corta, la osificación asociada al masetero presente alto desarrollo (Santana \& Dumont 2009), y la conexión entre el arco zigomático y el rostro se ubique anteriormente (Nogueira et al. 2005). Esta importancia funcional de los rasgos asociados a la mordida coincide con los valores más altos de similitud encontrados, que corresponden a los niveles más bajos de AF. Esto evidencia una relación entre la asimetría y la funcionalidad de ciertos rasgos. Los patrones de AF entre rasgos craneales se dan por las similitudes funcionales de los mismos, de forma que los niveles de AF van a estar correlacionados y se van a comportar como una unidad funcional (Gummer \& Brighman 1995). Por otra parte, la selección natural tiende a actuar de forma que se reduzca la $\mathrm{AF}$ en los rasgos que son funcionalmente más importantes (Crespi \& Vanderkist 1997).

El desarrollo de la asimetría fluctuante en ciertos caracteres no es independiente de su importancia funcional o de sus variaciones ontogenéticas; así, para que la hipótesis de la integración morfológica pueda extenderse al desarrollo de la AF, no solo debe considerarse la funcionalidad de los caracteres, sino las variaciones en sus procesos de desarrollo (Gummer \& Brigham 1995, Benítez \& Parra 2011).
Soulé (1967) mediante el estudio de la asimetría morfológica en Uta stansburiana (Squamata, Phrynosomatidae) planteó la hipótesis del Parámetro de Asimetría Poblacional, la cual plantea que dentro de una misma población todos los individuos presentan el mismo mecanismo homeostático para regular la influencia de factores ambientales en su desarrollo morfológico. En nuestro estudio, la correlación significativa entre las variables siempre fue positiva $y$ se mantuvo este patrón para todas las poblaciones de todas las localidades analizadas, por lo que el Parámetro de Asimetría establecido por Soulé (1967) no solo es significativo en $A$. lituratus a nivel individual, sino que puede ser extendido a nivel de población. Lo anterior sugiere que el mecanismo inherente que controla la ID actúa al interior de todas las poblaciones de la misma forma. Estos mismos resultados han sido reportados anteriormente para Eidolon belvum (Chiroptera, Pteropodidae) y Rousettus aegyptiacus (Chiroptera, Pteropodidae) en las islas del golfo de Guinea (Juste et al. 2001a).

El que las variables con AD presentaran alta similitud a nivel mandibular sugiere que aquella puede estar determinada principalmente por componentes genéticos y hereditarios; sin embargo, es poca la información que se conoce al respecto. Pélabon \& Hansen (2008) y Carter et al. (2009) sugirieron que la presencia de $\mathrm{AD}$ en rasgos específicos es una característica conservada en ciertos organismos, por lo que no es posible atribuirla a factores ambientales concretos, y sugieren que evolutivamente la herencia de la $\mathrm{AD}$ es una consecuencia de procesos acumulativos de selección natural.

\section{Diferencias entre sexos}

El que las hembras hayan presentado más AF en el esplacnocráneo, mientras los machos más en el neurocráneo, indica una presión funcional diferencial sobre la mordida. Esto puede estar evidenciando variaciones en el consumo de recursos entre sexos, posiblemente como resultado de diferencias en los requerimientos energéticos en la época reproductiva (Morrison 1978). Aunque aún no hay trabajos que describan las variaciones en la composición de la dieta con respecto al sexo o al estado reproductivo en especies del genero Artibeus (Herrera et al. 2001), sí se 
ha reportado que para las hembras de $A$. jamaicensis los costos energéticos derivados del forrajeo son mayores que para los machos (Morrison 1978). Se ha visto una acreción progresiva en la concentración de nitrógeno, calcio y minerales en la leche de las hembras de esta especie a lo largo del periodo de lactancia (Kwiecinski et al. 2003), lo que evidencia requerimientos nutricionales diferentes para las hembras que para los machos.

En los murciélagos frugívoros la complejidad en la morfología de los dientes (especialmente los molares) ha actuado como una respuesta a la presión adaptativa por optimizar la obtención del alimento, lo que ha resultado en un aumento de la importancia funcional de la dentición (Santana et al. 2011). El esplacnocráneo es una región que comprende huesos asociados a la dentición, por lo que una mayor AF para las hembras en esta región puede indicar que hay algún grado de diferenciación en la dieta entre sexos de esta especie, hipótesis que es necesario evaluar.

Otros estudios que han evaluado la $\mathrm{AF}$ en mamíferos muestran que las diferencias entre los sexos indican algún grado de dimorfismo sexual secundario a nivel craneal (Gannon et al. 1992). El dimorfismo sexual en murciélagos se ha relacionado con diferencias tróficas asociadas a la capacidad de carga durante el vuelo, la segregación de nichos y la fecundidad (Bornholt et al. 2008, Camargo \& de Oliveira 2012, Lisón et al. 2014).

La AF también ha mostrado relación con los procesos de selección sexual, los rasgos con una mayor importancia en la selección sexual tenderán a presentar menos asimetría, asegurando así un mayor éxito reproductivo en los individuos con rasgos más simétricos (Voigt et al. 2004). Esto se ha visto en especies de murciélagos que se organizan en harenes, donde los machos compiten por las hembras y el sitio de percha (Willig 1983). Se ha encontrado que $A$. lituratus presenta este comportamiento (Muñoz-Romo et al. 2008). Para conocer más a fondo estas diferencias es necesario obtener información adicional de las diferencias en el hábito alimentario entre machos y hembras de esta especie, así como de su comportamiento social, y analizar cómo estas variables pueden estar relacionadas con la asimetría.

\section{Conclusión}

La asimetría craneal en $A$. lituratus no es homogénea a lo largo de toda su estructura. Las regiones del cráneo altamente correlacionadas en su importancia funcional son más similares en sus niveles de asimetría fluctuante. El que las hembras presenten mayor asimetría fluctuante en el esplacnocráneo y los machos en el neurocráneo sugiere algún tipo de dimorfismo sexual secundario a nivel craneal en respuesta a diferencias en la importancia funcional de ciertos rasgos o debido a procesos de selección sexual.

\section{Agradecimientos}

Agradecemos a la Pontificia Universidad Javeriana y al Museo Javeriano de Historia Natural por el préstamo del material revisado para realizar este trabajo. A José A. Guerrero de la Universidad Autónoma del Estado de Morelos (UAEM), y a tres revisores anónimos por sus comentarios al manuscrito. El primer autor quiere agradecer a Laura Castañeda Gómez por sus comentarios y apoyo en la elaboración del manuscrito. Finalmente agradecemos al equipo del Laboratorio de Ecología Funcional por su apoyo y orientación durante el desarrollo de este proyecto.

\section{Conflictos de interés}

Los autores no tienen conflictos de interés de ningún tipo relacionados con este trabajo.

\section{Referencias}

Alberico M, Saavedra-R CA, García-Paredes H (2005) House bats of Cali, Colombia. Caldasia 27(1): 117-126

Benítez HA, Parra LE (2011) Asimetría fluctuante: una herramienta morfo-funcional para medir estabilidad del desarrollo. International Journal of Morphology 29: 14591469 doi: 10.4067/S0717-95022011000400066

Bornholt R, Oliveira LR, Fabián ME (2008) Sexual size dimorphism in Myotis nigricans (Schinz, 1821) (Chiroptera: Vespertilionidae) from south Brazil. Brazilian Journal of Biology 68(4): 897-904

Calonge B, Vela-Vargas I, Pérez-Torres J (2010) Murciélagos asociados a una finca ganadera en Córdoba (Colombia). MVZ Córdoba 15(1): 1938-1943 
Camargo NFd, de Oliveira HF (2012) Sexual Dimorphism in Sturnira lilium (Chiroptera, Phyllostomidae): Can pregnancy and pup carrying be reponsible for differences in wing shape?. PLoS ONE 7(11): e49734 doi: 10.1371 /journal.pone.0049734

Carter AJ, Osborne E, Houle D (2009) Heritability of directional asymmetry in Drosophila melanogaster. International Journal of Evolutionary Biology 1-7 doi: 10.4061/2009/759159

Crespi BJ, Vanderkist BA (1997) Fluctuating asymmetry in vestigial and functional traits of a haplodiploid insect. Heredity 79: 624-630 doi: 10.1038/hdy.1997.208

Estrada-Villegas S, Pérez-Torres J, Stevenson PR (2010) Ensamblaje de murciélagos en un bosque subandino colombiano y análisis sobre la dieta de algunas especies. Mastozoología Neotropical 17(1): 31-41

Galindo JG (2004) Clasificación de los murciélagos de la región de Los Tuxtlas, Veracruz, respecto a su respuesta a la fragmentación del hábitat. Acta Zoológica Mexicana (Nueva Serie): 239-243

Gannon MR, Willig MR, Jones JK (1992) Morphometric variation, measurement error, and fluctuating asymmetry in the red fig-eating bat (Stenoderma rufum). Texas Journal of Science 44: 389-404

Graham JH, Freeman DC, Emlen JM (1993a) Developmental stability: a sensitive indicator of populations under stress. In: Landis WG, Hughes JS, Lewis MA, (eds) Environmental Toxicology and Risk Assessment. American Society for Testing and Materials, Philadelphia. Pp 136-158

Graham JH, Freeman DC, Emlen JM (1993b) Antisymmetry, directional asymmetry, and dynamic morphogenesis. Genetica 89(1-3): 121-137

Graham JH, Raz S, Hel-Or H, Nevo E (2010) Fluctuating asymmetry: methods, theory, and applications. Symmetry 2(2): 466-540 doi: 10.3390/sym2020466

Gummer DL, Brigham RM (1995) Does fluctuating asymmetry reflect the importance of traits in little brown bats (Myotis lucifugus)? Canadian Journal of Zoology 73(5): 990-992 doi: 10.1139/z95-116

Habel JC, Reuter M, Dress C, Pfaender J (2012) Does isolation affect phenotypic variability and fluctuating asymmetry in the endangered Red Apollo? Journal of Insect Conservation 16: 571-579 doi: 10.1007/s10841011-9442-3

Hallgrímsson B (1998) Fluctuating asymmetry in the mammalian skeleton: evolutionary and developmental implications. Evolutionary Biology 30: 187-251 doi: 10.1007/978-1-4899-1751-5_6

Hammer A, Harper DA, Ryan PD (2001) PAST (versión 2.17)
HerreraLG, Hobson KA, Manzo AA, Estrada DB, SánchezCordero DB, Méndez GC (2001) The role of fruits and insects in the nutrition of frugivorous bats: evaluating the use of stable isotope models. Biotropica 33(3): 520528 doi: 10.1111/j.1744-7429.2001.tb00206.x

Juste J, López-González C, Strauss RE (2001a) Analysis of asymmetries in the African fruit bats Eidolon helvum and Rousettus egyptiacus (Mammalia: Megachiroptera) from the islands of the Gulf of Guinea. II. Integration and levels of multivariate fluctuating asymmetry across a geographical range. Journal of Evolutionary Biology 14: 672-680 doi: 10.1046/j.1420-9101.2001.00299.x

Juste J, López-González C, Strauss RE (2001b) Analysis of asymmetries in the African fruit bats Eidolon helvum and Rousettus egyptiacus (Mammalia : Megachiroptera) from the islands of the Gulf of Guinea . I. Variance and size components of bilateral variation. Journal of Evolutionary Biology 14: 663-671 doi: 10.1046/j.14209101.2001.00298.x

Kalko EK, Handley CE (1994) Evolution, biogeography, and description of a new species of fruit-eating bat, genus Artibeus Leach (1821), from Panamá. Zeitschrift für Sängetierkunde 59(5): 257-273

Kellner JR, Alford RA (2003) The ontogeny of fluctuating asymmetry. The American Naturalist 161(6): 931-946 doi: $10.1086 / 375177$

Klingenberg CP (2008) Morphological integration and developmental modularity. Annual Review of Ecology Evolution and Systematics 39: 115-132 doi: 10.1146/ annurev.ecolsys.37.091305.110054

Knierim U, Van Dongen S, Forkman B, Tuyttens FM, Spinka M, et al. (2007) Fluctuating asymmetry as an animal welfare indicator - Aa review of methodology and validity. Physiology and Behavior 92(3): 398-421 doi: 10.1016/j.physbeh.2007.02.014

Kwiecinski GG, Falzone M, Studier EH (2003) Milk concentration and postnatal accretion of minerals and nitrogen in two phyllostomid bats. Journal of Mammalogy 84(3): 926-936

Leamy LJ (1977) Genetic and environmental correlations of morphometric traits in random bred house mice. Evolution 31: 357-369

Lisón F, Haz Á, González-Revelles C, Calvo JF (2014) Sexual size dimorphism in greater mouse-eared bat Myotis myotis (Chiroptera: Vespertilionidae) from a mediterranean region. Acta Zoologica (Stockholm) 95: 137-143 doi: 10.1111/azo.12012

Marchán-Rivedeneira MR, Larsen PA, Phillips CJ, Strauss R, Baker RJ (2012) On the association between environmental gradients and skull size variation in the great fruit-eating bat, Artibeus lituratus (Chiroptera: Phyllostomidae). Biological Journal of the Linnean Society 105: 623-634 doi: 10.1111/j.1095-8312.2011.01804.x 
Mateos C, Alarcos S, Carranza J, Sánchez-Prieto CB, Valencia J (2008) Fluctuating asymmetry of red deer antlers negatively relates to individual condition and proximity to prime age. Animal Behaviour 75(5): 16291640 doi: 10.1016/j.anbehav.2007.10.016

Medellín RA, Equihua M, Amin MA (2000) Bat diversity and abundance as indicators of disturbance in neotropical rainforests. Conservation Biology 14(6): 1666-1675

Messier S, Mitton JB (1996) Heterozygosity at the malate dehydrogenase locus and developmental homeostasis in Apis mellifera. Heredity 76: 616-622 doi: 10.1038/ hdy. 1996.88

Morrison DW (1978) Foraging ecology and energetics of the frugivorous bat Artibeus Jamaicensis. Ecology 59(4): 716-723

Muñoz-Romo M, Herrera ME, Kunz TH (2008) Roosting behavior and group stability of the big fruit-eating bat Artibeus lituratus (Chiroptera: Phyllostomidae). Mammalian Biology 73: 214-221 doi: 10.1016/j. mambio.2007.05.013

Naugler CT, Ludman MD (1996) Fluctuating asymmetry and disorders of developmental origin. American Journal of MedicalGenetics 66(1):15-20 doi:10.1002/(SICI)10968628(19961202)66:1<15::AID-AJMG4>3.0.CO;2-V

Nogueira MR, Monteiro LR, Peracchi AL, Araújo AF (2005) Ecomorphological analysis of the masticatory apparatus in the seed-eating bats, genus Chiroderma (Chiroptera: Phyllostomidae). Journal of Zoology 266: 355-364 doi: 10.1017/S0952836905007053

Ortegón-Martínez D, Pérez-Torres J (2007) Structure and composition of the bat assemblage associate to a shade coffee plantation in Mesa de los Santos (Santander), Colombia. Actualidades Biológicas 29(87): 215-228

Pélabon C, Hansen TF (2008) On the adaptive accuracy of directional asymmetry in insect wing size. Evolution 62(11): 2855-2867 doi: 10.1111/j.15585646.2008.00495.x

Richardson L, Loomis J (2009) The total economic value of threatened, endangered and rare species: an updated meta-analysis. Ecological Economics 68(5): 1535-1548 doi: 10.1016/j.ecolecon.2008.10.016

Rohlf FJ (2013) TpsDig2 (Versión 2.17)

Santana SE, Dumont ER (2009) Connecting behaviour and performance: the evolution of biting behaviour and bite performance in bats. Journal of Evolutionary Biology 25: 839-847 doi: 10.1111/j.1420-9101.2009.01827.x

Santana SE, Strait S, Dumont ER (2011) The better to eat you with: functional correlates of toothstructure in bats. Functional Ecology 25: 839-847 doi: 10.1111/j.13652435.2011.01832.x
Sonne C, Riget FF, Dietz R, Kirkegaard M, Born EW, et al. (2005) Trends in fluctuating asymmetry in East Greenland polar bears (Ursus maritimus) from 1892 to 2002 in relation to organohalogen pollution. The Science of the Total Environment 341(1-3): 81-96

Soulé M (1967) Phenetics of natural populations. II. Asymmetry and evolution in a lizard. The American Naturalist 101: 141-160

Soulé ME, Baker B (1968) Phenetics of natural populations. IV. The population asymmetry parameter in the butterfly Coenonympha tullia. Heredity 23: 611-614

Teixeira CP, Hirsch A, Perini H, Young R (2006) Marsupials from space: fluctuating asymmetry, geographical information systems and animal conservation. Proceedings of the Royal Society Series B 273: 1007-1012 doi: 10.1098/rspb.2005.3386

Van Dongen S, Sprengers SE, Löfstedt C, Matthysen E (1999) Heritability of tibia fluctuating asymmetry and developmental instability in the winter moth (Operophtera brumata L.) (Lepidoptera, Geometridae). Heredity 82: 535-542 doi: 10.1038/sj.hdy.6885230

Voigt CC, Heckel G, Mayer F (2004) Sexual selection favours small and symmetric males in the polygynous greater sac-winged bat Saccopteryx bilineata (Emballonuridae, Chiroptera). Behavioral Ecology and Sociobiology 57(5): 457-464 doi: 10.1007/s00265-004-0874-6

Willig MR (1983) Composition, microgeographic variation, and sexual dimorphism in Caatingas and Cerrado bat communities from northeast Brazil. Bulletin of Carnegie Museum of Natural History 23: 1-131

Windig JJ, Nylin S (2000) How to compare fluctuating asymmetry of different traits. Journal of Evolutionary Biology 13: 29-37

Zachos FE, Hartl GB, Suchentrunk F (2007) Fluctuating asymmetry and genetic variability in the roe deer (Capreolus capreolus): a test of the developmental stability hypothesis in mammals using neutral molecular markers. Heredity 98: 392-400 doi: 10.1038/sj.hdy.6800954 
Asimetría cráneo-mandibular de Artibeus lituratus (Chiroptera, Phyllostomidae) en Colombia

Resumen. Analizar la asimetría en especies asociadas a ambientes disturbados permite evaluar la plasticidad morfológica de especies generalistas, y las diferencias evolutivas entre sexos y poblaciones en la respuesta al estrés ambiental y genético. Este trabajo evalúa la asimetría craneal de Artibeus lituratus en Colombia. Esta especie tiene distribución amplia y alta abundancia, pero se desconoce su plasticidad morfológica. Se caracterizó la presencia y patrones de asimetría fluctuante, asimetría direccional y antisimetría. Se tomaron once medidas craneométricas de 146 individuos de diferentes regiones geográficas. Se encontró significancia para asimetría fluctuante en todas las medidas, en tres para asimetría direccional y antisimetría, y no se encontró significancia para el error de medida. Las hembras presentaron más asimetría fluctuante en el esplacnocráneo y los machos en el neurocráneo. Se evidenció menor asimetría y mayor similitud en variables con importancia funcional en la mordida. Los rasgos con asimetría direccional presentaron asociación a nivel mandibular. Se discute en qué medida la presencia y los niveles de asimetría craneal se relacionan con la similitud funcional que tengan diferentes rasgos.

Palabras clave: Stenodermatinae; ecomorfología; craneometría; parámetro de asimetría; Neotrópico
Assimetria crânio-mandibular em Artibeus lituratus (Chiroptera, Phyllostomidae) na Colômbia

Resumo. Analisar a assimetria em espécies associadas a ambientes perturbados permite avaliar a plasticidade morfológica de espécies generalistas e as diferenças evolutivas entre sexos na resposta ao estresse ambiental e genético. Este trabalho avalia a assimetria cranial de Artibeus lituratus na Colômbia. Esta espécie tem distribuição ampla e é extremamente abundante, mas desconhece-se a sua plasticidade morfológica. Caracterizou-se a presença e padrões nos níveis de assimetria flutuantes, assimetria direcional e anti-simetria. Foram obtidas onze medidas craniométricas de 146 indivíduos de diferentes regiões geográficas. Encontrou-se significância para assimetria flutuante em todas as medidas, em três para assimetria direcional e anti-simetria, e não se encontrou significância para o erro de medida. As fêmeas apresentaram maior assimetria flutuante no esplacnocrânio, enquanto nos machos essa ocorreu no neurocrânio. Evidenciou-se menor assimetria e maior similaridade em variáveis com maior importância funcional na mordida. Os caracteres com assimetria direcional apresentaram maior associação com a mandíbula. Discute-se em que medida a presença e os níveis de assimetria cranial se relacionam com a similaridade funcional dos diferentes caracteres.

Palavras-chave: Stenodermatinae; ecomorfologia; craniometria; parâmetro de assimetria; Neotrópicos 